\title{
LIMITING CROSSING PROBABILITIES OF RANDOM FIELDS
}

\author{
L. PEREIRA* AND \\ H. FERREIRA, ${ }^{* *}$ University of Beira Interior
}

\begin{abstract}
Random fields on $\mathbb{Z}_{+}^{2}$, with long-range weak dependence for each coordinate individually, usually present clustering of high values. For each one of the eight directions in $\mathbb{Z}_{+}^{2}$, we formulate restriction conditions on local occurrence of two or more crossings of high levels. These smooth oscillation conditions enable computation of the extremal index as a clustering measure from the limiting mean number of crossings. In fact, only four directions must be inspected since for opposite directions we find the same local path crossing behaviour and the same limiting mean number of crossings. The general theory is illustrated with several 1-dependent nonstationary random fields.
\end{abstract}

Keywords: Random field; dependence; nonstationarity; extremal index

2000 Mathematics Subject Classification: Primary 60G60; 60G70; 60F99

\section{Introduction}

Let $\boldsymbol{X}=\left\{X_{\boldsymbol{n}}\right\}_{\boldsymbol{n} \geq \mathbf{1}}$ be a random field on $\mathbb{Z}_{+}^{d}$, where $\mathbb{Z}_{+}$is the set of all positive integers and $d \geq 2$. We shall consider the conditions and results for $d=2$ since it is notationally simpler and the proofs for higher dimensions follow by analogous arguments. The inequality $\boldsymbol{i} \leq \boldsymbol{n}$ means $i_{k} \leq n_{k}, k=1,2$.

For a family of real levels $\left\{u_{n, i}: \boldsymbol{i} \leq \boldsymbol{n}\right\}_{\boldsymbol{n} \geq \mathbf{1}}$ and a subset $I$ of the rectangle of points $R_{n}=\left\{1, \ldots, n_{1}\right\} \times\left\{1, \ldots, n_{2}\right\}$, we will denote the event $\left\{\bigcap_{i \in I} X_{i} \leq u_{n, i}\right\}$ by $\left\{M_{n}(I) \leq u\right\}$, or more simply by $\left\{M_{n} \leq u\right\}$ when $I=R_{n}$.

For each $i=1,2$, we say that the pair $I \subset \mathbb{Z}_{+}^{2}$ and $J \subset \mathbb{Z}_{+}^{2}$ is in $\delta_{i}(l)$ if the distance between $\Pi_{i}(I)$ and $\Pi_{i}(J)$ is greater than or equal to $l$, where $\Pi_{i}, i=1,2$, denotes the cartesian projection. The distance $d(I, J)$ between sets $I$ and $J$ of $\mathbb{Z}_{+}^{d}, d \geq 1$, is the minimum of the distances $d(\boldsymbol{i}, \boldsymbol{j})=\max \left\{\left|i_{s}-j_{s}\right|, s=1, \ldots, d\right\}, \boldsymbol{i} \in I$ and $\boldsymbol{j} \in J$.

Suppose that $\boldsymbol{X}$ satisfies a coordinatewise-mixing-type condition, such as the $\Delta\left(u_{n}\right)$-condition introduced in [9] which exploits the past and future separation one coordinate at a time. Let $\mathcal{F}$ be a family of index sets in $R_{\boldsymbol{n}}$. We shall assume that there are sequences of integer-valued constants $\left\{k_{n_{i}}\right\}$ and $\left\{l_{n_{i}}\right\}, i=1,2$, such that, as $\boldsymbol{n}=\left(n_{1}, n_{2}\right) \rightarrow \infty$,

$$
\left(k_{n_{1}}, k_{n_{2}}\right) \rightarrow \infty, \quad\left(\frac{k_{n_{1}} l_{n_{1}}}{n_{1}}, \frac{k_{n_{2}} l_{n_{2}}}{n_{2}}\right) \rightarrow \mathbf{0},
$$

Received 6 December 2005; revision received 26 February 2006.

* Postal address: Department of Mathematics, University of Beira Interior, 6200 Covilhã, Portugal.

** Email address: helena@mat.ubi.pt 
and $\left(k_{n_{1}} \Delta_{1}, k_{n_{1}} k_{n_{2}} \Delta_{2}\right) \rightarrow \boldsymbol{0}$. Here, $\Delta_{i}$ are the components of the mixing coefficient defined by

$$
\Delta_{1}=\sup \left|\mathrm{P}\left(M_{\boldsymbol{n}}\left(I_{1}\right) \leq u, M_{\boldsymbol{n}}\left(I_{2}\right) \leq u\right)-\mathrm{P}\left(M_{\boldsymbol{n}}\left(I_{1}\right) \leq u\right) \mathrm{P}\left(M_{\boldsymbol{n}}\left(I_{2}\right) \leq u\right)\right|,
$$

where the supremum is taken over pairs $I_{1}$ and $I_{2}$ in $\S_{1}\left(l_{n_{1}}\right) \cap \mathcal{F}$, and

$$
\Delta_{2}=\sup \left|\mathrm{P}\left(M_{n}\left(I_{1}\right) \leq u, M_{n}\left(I_{2}\right) \leq u\right)-\mathrm{P}\left(M_{n}\left(I_{1}\right) \leq u\right) \mathrm{P}\left(M_{n}\left(I_{2}\right) \leq u\right)\right|,
$$

where the supremum is taken over pairs $I_{1}$ and $I_{2}$ in $\delta_{2}\left(l_{n_{2}}\right) \cap \mathcal{F}$. We then say that $\boldsymbol{X}$ satisfies the $D\left(u_{n, i}\right)$-condition over $\mathcal{F}$. In fact, we could consider a slightly weaker condition, as in [9], if we were concerned only with stationary random fields.

In Section 2, we prove that the maxima over disjoint rectangles behave asymptotically as independent maxima.

Restrictions on clustering of high values for stationary and nonstationary time series have been considered in the form of the $D^{\prime}$-condition introduced in [7]; see also [5]. In [11], the authors introduced a $D^{\prime}$-condition tailored for random fields which are not necessarily stationary. That condition and the coordinatewise long-range dependence lead to a Poisson approximation for the probability of no exceedances over $R_{n}$; this result can be applied to nonstationary Gaussian random fields.

In Section 3, we discuss the behaviour of the maxima when clustering of high values of $\boldsymbol{X}$ is allowed, but we restrict the local occurrence of two or more crossings of the high levels $u_{n, i}$. For each one of the eight directions in $\mathbb{Z}_{+}^{2}$, we can restrict the local occurrence of two or more crossings. These smooth oscillation conditions enable us to compute a clustering measure, called the extremal index, from the limiting mean number of crossings. We prove that, in fact, only four directions must be inspected since for opposite directions we find the same local path crossing behaviour and the same limiting mean number of crossings.

We illustrate these results with several 1-dependent nonstationary random fields, which satisfy different local crossing conditions.

\section{Asymptotic independence of maxima}

Under the coordinatewise-mixing $D\left(u_{n, i}\right)$-condition, we have asymptotic independence of maxima over disjoint rectangles of indexes. In the following, $\bar{F}_{\max }$ denotes

$$
\max \left\{\mathrm{P}\left(X_{\boldsymbol{i}}>u_{\boldsymbol{n}, \boldsymbol{i}}\right): \boldsymbol{i} \leq \boldsymbol{n}\right\} .
$$

Proposition 2.1. Suppose that the random field $\boldsymbol{X}$ satisfies the $D\left(u_{n, i}\right)$-condition over $\mathcal{F}$ such that $(I \subset J \wedge J \in \mathcal{F}) \Longrightarrow I \in \mathcal{F}$ and for $\left\{u_{\mathbf{n}, \mathbf{i}}: \boldsymbol{i} \leq \boldsymbol{n}\right\}_{\boldsymbol{n} \geq \mathbf{1}}$ such that

$$
\left\{n_{1} n_{2} \bar{F}_{\max }\right\}_{\boldsymbol{n} \geq \mathbf{1}} \text { is bounded. }
$$

If $\boldsymbol{V}_{r, p}=I_{r} \times J_{r, p}, r=1, \ldots, k_{n_{1}}, p=1, \ldots, k_{n_{2}}$, are disjoint rectangles in $\mathcal{F}$, then, as $\boldsymbol{n} \rightarrow \infty$,

$$
\mathrm{P}\left(\bigcap_{r, p} M_{\boldsymbol{n}}\left(\boldsymbol{V}_{r, p}\right) \leq u\right)-\prod_{r, p} \mathrm{P}\left(M_{\boldsymbol{n}}\left(\boldsymbol{V}_{r, p}\right) \leq u\right) \rightarrow 0 .
$$

Proof. From (1.1) and (2.1), for the purpose of the above convergence we can assume that $\Pi_{1}\left(\boldsymbol{V}_{r, p}\right)>l_{n_{1}}$ or $\Pi_{2}\left(\boldsymbol{V}_{r, p}\right)>l_{n_{2}}$. If all the pairs of rectangles $\boldsymbol{V}_{r, p}$ are in $\varsigma_{1}\left(l_{n_{1}}\right) \cup \wp_{2}\left(l_{n_{2}}\right)$ then the result follows inductively from the $D\left(u_{n, i}\right)$-condition. On the contrary, we can eliminate $l_{n_{1}}$ columns or $l_{n_{2}}$ rows of indices in $\boldsymbol{V}_{r, p}$ in order to obtain $\boldsymbol{V}_{r, p}^{*} \subset \boldsymbol{V}_{r, p}, r=1, \ldots, k_{n_{1}}$, $p=1, \ldots, k_{n_{2}}$, to which we can inductively apply the $D\left(u_{n, i}\right)$-condition. 


\section{Limiting crossing probabilities}

We now discuss the limiting distribution of the maximum when, in addition to the coordinatewise-mixing condition, we restrict the local path behaviour with respect to the number of crossings of the high levels $u_{n, i}$.

Since the natural notion of crossing at $\boldsymbol{i}=\left(i_{1}, i_{2}\right)$ would take into consideration the values of the random field over the eight neighbours of $\boldsymbol{i}$, i.e. over the points $\boldsymbol{j}$ such that $d(\boldsymbol{i}, \boldsymbol{j})=1$, by taking $\beta(\{\boldsymbol{i}\})=\{\boldsymbol{j}: d(\boldsymbol{i}, \boldsymbol{j})=1\}$, we say that $\boldsymbol{X}$ has a crossing at $\boldsymbol{i}$ if the event

$$
B_{i, n}=\left\{X_{i} \leq u_{n, i}, \bigcup_{j \in \beta(\{i\})} X_{j}>u_{n, j}\right\}
$$

occurs.

Using the ideas of [8], in combination with [3] and [6], to avoid clustering of crossings by a nonstationary random field, we could be led to suspect that, for each rectangle $I$ satisfying

$$
\sum_{\boldsymbol{i} \in I} \mathrm{P}\left(B_{\boldsymbol{i}, \boldsymbol{n}}\right) \leq \frac{1}{k_{n_{1}} k_{n_{2}}} \sum_{\boldsymbol{i} \leq \boldsymbol{n}} \mathrm{P}\left(B_{\boldsymbol{i}, \boldsymbol{n}}\right),
$$

the following condition holds:

$$
k_{n_{1}} k_{n_{2}} \sum_{\boldsymbol{i}, \boldsymbol{j} \in I} \mathrm{P}\left(B_{\boldsymbol{i}, \boldsymbol{n}}, B_{\boldsymbol{j}, \boldsymbol{n}}\right) \stackrel{\boldsymbol{n} \rightarrow \infty}{\longrightarrow} 0 .
$$

However, we will show that an independent and identically distributed (i.i.d.) random field does not satisfy (3.1) for normalized levels $\left\{u_{n}\right\}_{n \geq 1}$ such that

$$
n_{1} n_{2} \mathrm{P}\left(X_{1}>u_{n}\right) \stackrel{n \rightarrow \infty}{\longrightarrow} \tau \text {. }
$$

For each $\boldsymbol{i}=\left(i_{1}, i_{2}\right) \in \mathbb{Z}_{+}^{2}$, let $b_{s}(\boldsymbol{i}), s=1, \ldots, 8$, be the neighbours of $\boldsymbol{i}$ defined as follows:

$$
\begin{array}{ll}
b_{1}(\boldsymbol{i})=\left(i_{1}+1, i_{2}\right), & b_{2}(\boldsymbol{i})=\boldsymbol{i}+\mathbf{1}, \\
b_{3}(\boldsymbol{i})=\left(i_{1}, i_{2}+1\right), & b_{4}(\boldsymbol{i})=\left(i_{1}-1, i_{2}+1\right), \\
b_{5}(\boldsymbol{i})=\left(i_{1}-1, i_{2}\right), & b_{6}(\boldsymbol{i})=\boldsymbol{i}-\mathbf{1}, \\
b_{7}(\boldsymbol{i})=\left(i_{1}, i_{2}-1\right), & b_{8}(\boldsymbol{i})=\left(i_{1}+1, i_{2}-1\right) .
\end{array}
$$

For each $s=1, \ldots, 8$, we shall denote the $s$-crossing event $\left\{X_{i} \leq u_{n, i}, X_{b_{s}(i)}>u_{n, b_{s}(i)}\right\}$ by $B_{i, b_{s}(i), n}$, or more simply by $B_{i, b_{s}(i)}$, where $X_{b_{s}(i)}=-\infty$ if $b_{s}(i) \notin \mathbb{Z}_{+}^{2}$.

In fact, for an i.i.d. random field $\boldsymbol{X},\left\{u_{\boldsymbol{n}}\right\}_{\boldsymbol{n} \geq \mathbf{1}}$ satisfying (3.2), and $I=\left\{1, \ldots,\left\lfloor n_{1} / k_{n_{1}}\right\rfloor\right\} \times$ $\left\{1, \ldots,\left\lfloor n_{2} / k_{n_{2}}\right\rfloor\right\}$ (where $\lfloor\cdot\rfloor$ denotes the integer-part function), we have

$$
\sum_{\boldsymbol{i} \in I} \mathrm{P}\left(B_{\boldsymbol{i}, n}\right) \leq \frac{1}{k_{n_{1}} k_{n_{2}}} \sum_{\boldsymbol{i} \leq \boldsymbol{n}} \mathrm{P}\left(B_{\boldsymbol{i}, n}\right)
$$

and

$$
\begin{aligned}
k_{n_{1}} k_{n_{2}} \sum_{\boldsymbol{i}, \boldsymbol{j} \in I} \mathrm{P}\left(B_{\boldsymbol{i}, n}, B_{\boldsymbol{j}, n}\right) & \geq k_{n_{1}} k_{n_{2}} \sum_{s \neq t} \sum_{\boldsymbol{i}, \boldsymbol{j} \in I} \mathrm{P}\left(B_{\boldsymbol{i}, b_{s}(\boldsymbol{i})}, B_{\boldsymbol{j}, b_{t}(\boldsymbol{j})}\right) \\
& \geq k_{n_{1}} k_{n_{2}} \prod_{i=1}^{2}\left(\left\lfloor\frac{n_{i}}{k_{n_{i}}}\right\rfloor-1\right) \mathrm{P}\left(X_{\mathbf{1}}>u_{\boldsymbol{n}}\right) \mathrm{P}^{2}\left(X_{\mathbf{1}} \leq u_{\boldsymbol{n}}\right),
\end{aligned}
$$


which tends to $\tau$ as $\boldsymbol{n} \rightarrow \infty$. By analogous reasoning with subsets of $\beta(\{\boldsymbol{i}\})$ with more than one element, we conclude that this kind of restriction on more than one direction is not satisfied by i.i.d. random fields and normalized levels.

Since the only direction that uses the notion of past and future along both coordinate axes simultaneously is the diagonal direction from $\boldsymbol{i}$ to $\boldsymbol{i}+\mathbf{1}$, the authors considered in [11] a condition which restricts the local occurrence of two or more of these diagonal crossings, i.e. a condition that restricts the local occurrence of two or more events $\left\{X_{i} \leq u_{n, i}, X_{i+1}>u_{n, i+1}\right\}$.

Here we shall consider a more general approach to crossing events of random fields using a family of eight local conditions. Different examples can verify different conditions of this family, as we shall illustrate in Section 4.

Let $\mathcal{C}\left(B_{i, b_{s}(i), n}\right)$ denote the family of index sets $I \subset R_{n}$ such that

$$
\sum_{i \in I} \mathrm{P}\left(B_{i, b_{s}(i), n}\right) \leq \frac{1}{k_{n_{1}} k_{n_{2}}} \sum_{i \leq n} \mathrm{P}\left(B_{i, b_{s}(i), n}\right) .
$$

Definition 3.1. Let $s \in\{1, \ldots, 8\}$. The $D^{\prime \prime}\left(B_{i, b_{s}(i), n}\right)$-condition holds for $\boldsymbol{X}$ if, for each

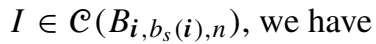

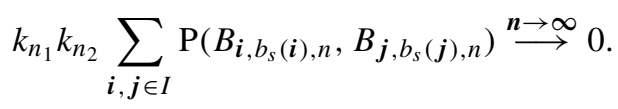

We show in Proposition 3.2, below, that each one of these eight local $D^{\prime \prime}\left(B_{i, b_{s}(i), n}\right)$ conditions is a sufficient condition to compute the limit of $\mathrm{P}\left(M_{\boldsymbol{n}} \leq u\right)$ from the limiting mean number of $s$-crossings. In order to apply Proposition 3.2, we only need to inspect one of four directions, as the next result shows.

Proposition 3.1. Suppose that the random field $\boldsymbol{X}$ satisfies (2.1) and let $s \in\{1, \ldots, 8\}$. Then

(i) $\boldsymbol{X}$ satisfies the $D^{\prime \prime}\left(B_{i, b_{s}(i), n}\right)$-condition if and only if it satisfies the $D^{\prime \prime}\left(B_{i, \overline{b_{s}}}(\boldsymbol{i}), n\right)$ condition, where $\overline{b_{s}}=b_{s+4}$,

(ii) $\sum_{i \leq n} \mathrm{P}\left(B_{i, b_{s}(i), n}\right) \stackrel{n \rightarrow \infty}{\longrightarrow} v>0$ if and only if $\sum_{\boldsymbol{i} \leq \boldsymbol{n}} \mathrm{P}\left(B_{\boldsymbol{i}, \overline{b_{s}}}(\boldsymbol{i}), n\right) \stackrel{n \rightarrow \infty}{\longrightarrow} v>0$.

Proof. To obtain part (i) we first note that

$$
\begin{aligned}
& \sum_{\boldsymbol{i}, \boldsymbol{j} \in I} \mathrm{P}\left(B_{\boldsymbol{i}, b_{s}(\boldsymbol{i})}, B_{\boldsymbol{j}, b_{s}(\boldsymbol{j})}\right) \\
& =\sum_{\boldsymbol{i}, \boldsymbol{j} \in I} \mathrm{P}\left(B_{\boldsymbol{i}, b_{s}(\boldsymbol{i})}, X_{b_{s}(\boldsymbol{j})}>u_{n, b_{s}(\boldsymbol{j})}\right)-\sum_{\boldsymbol{i}, \boldsymbol{j} \in I} \mathrm{P}\left(B_{\left.\boldsymbol{i}, b_{s}(\boldsymbol{i}), X_{\boldsymbol{j}}>u_{n, \boldsymbol{j}}\right)}\right. \\
& \quad+\sum_{\boldsymbol{i}, \boldsymbol{j} \in I} \mathrm{P}\left(B_{\boldsymbol{i}, b_{s}(\boldsymbol{i})}, X_{\boldsymbol{j}}>u_{n, \boldsymbol{j}}, X_{b_{s}(\boldsymbol{j})} \leq u_{n, b_{s}(\boldsymbol{j})}\right)
\end{aligned}
$$

By applying the same decomposition to $B_{i, b_{s}(i)}$ in each of the above terms, we obtain

$$
\sum_{\boldsymbol{i}, \boldsymbol{j} \in I} \mathrm{P}\left(B_{\boldsymbol{i}, b_{s}(\boldsymbol{i})}, B_{\boldsymbol{j}, b_{s}(\boldsymbol{j})}\right)=\sum_{\boldsymbol{i}, \boldsymbol{j} \in I} \mathrm{P}\left(B_{\boldsymbol{i}, \overline{b_{s}}(\boldsymbol{i})}, B_{\boldsymbol{j}, \overline{b_{s}}(\boldsymbol{j})}\right)+o\left(k_{n_{1}} k_{n_{2}}\right) .
$$

Part (ii) follows by an analogous argument. 
Under the conditions (1.1) and (2.1), in the proof of Proposition 3.2, below, we can suppose that, for each rectangle $V_{r, p}$ in the partitions that arise for $R_{n}$, the variables $X_{i}$ with indices in the boundary of $\boldsymbol{V}_{r, p}$ exhibit values below the corresponding levels $u_{n, i}$. Asymptotically, the probability of the complement of that event is negligible. So, for each fixed $s$, some event $B_{i, b_{s}(i)}$ with $\boldsymbol{i} \in \boldsymbol{V}_{r, p}$ occurs if and only if we have some exceedance over $\boldsymbol{V}_{r, p}$.

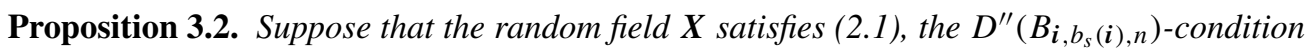
holds for some $s \in\{1, \ldots, 8\}$, and the $D\left(u_{n, i}\right)$-condition holds over $\mathcal{C}\left(B_{i, b_{s}}(i), n\right)$. Then, as $\boldsymbol{n} \rightarrow \infty$,

$$
\mathrm{P}\left(\bigcap_{\boldsymbol{i} \leq \boldsymbol{n}} X_{i} \leq u_{n, i}\right) \rightarrow \mathrm{e}^{-v}, \quad v>0,
$$

if and only if

$$
\sum_{i \leq n} \mathrm{P}\left(B_{i, b_{s}(i), n}\right) \rightarrow v>0 .
$$

Proof. Let $\left\{k_{n_{i}}\right\}, i=1,2$, as in (1.1). We will build $k_{n_{1}} k_{n_{2}}$ rectangles in $\mathcal{C}\left(B_{i, b_{s}(i), n}\right)$ as follows. First split $R_{\boldsymbol{n}}$ into $k_{n_{1}}$ quasi-rectangles

$$
\begin{aligned}
I_{r}^{\prime}= & \left(\left\{s_{r-1}+1\right\} \times\left\{t_{r-1}^{*}+1, \ldots, n_{2}\right\}\right) \cup\left(\left\{s_{r-1}+2, \ldots, s_{r}\right\} \times\left\{1, \ldots, n_{2}\right\}\right) \\
& \cup\left(\left\{s_{r}+1\right\} \times\left\{1, \ldots, t_{r}^{*} \leq n_{2}\right\}\right), \quad r=0, \ldots, k_{n_{1}}, s_{0}=0=t_{0}^{*},
\end{aligned}
$$

with $t_{r}^{*}$ maximally chosen such that

$$
\sum_{\boldsymbol{i} \in I_{r}^{\prime}} \mathrm{P}\left(B_{\boldsymbol{i}, b_{s}(\boldsymbol{i})}\right) \leq \frac{1}{k_{n_{1}}} \sum_{\boldsymbol{i} \leq \boldsymbol{n}} \mathrm{P}\left(B_{\boldsymbol{i}, b_{s}(\boldsymbol{i})}\right) .
$$

Let $I_{r}=\left\{s_{r-1}+2, \ldots, s_{r}\right\} \times\left\{1, \ldots, n_{2}\right\}$, and now split each rectangle $I_{r}$ into $k_{n_{1}} k_{n_{2}}$ quasirectangles

$$
\begin{aligned}
\boldsymbol{V}_{r, p}^{\prime}= & \left(\left\{s_{r, p-1}^{*}+1, \ldots, s_{r}\right\} \times\left\{t_{p-1}+1\right\}\right) \cup\left(\left\{s_{r-1}+1, \ldots, s_{r}\right\} \times\left\{t_{p-1}+2, \ldots, t_{p}\right\}\right) \\
& \cup\left(\left\{s_{r-1}+1, \ldots, s_{r, p}^{*} \leq s_{r}\right\} \times\left\{t_{p}+1\right\}\right), \quad p=1, \ldots, k_{n_{2}}, t_{0}=0, s_{r, 0}^{*}=s_{r-1},
\end{aligned}
$$

with $s_{r, p}^{*}$ maximally chosen such that

$$
\sum_{\boldsymbol{i} \in \boldsymbol{V}_{r, p}^{\prime}} \mathrm{P}\left(B_{\boldsymbol{i}, b_{s}(\boldsymbol{i})}\right) \leq \frac{1}{k_{n_{1}} k_{n_{2}}} \sum_{\boldsymbol{i} \leq \boldsymbol{n}} P\left(B_{\boldsymbol{i}, b_{s}(\boldsymbol{i})}\right) .
$$

Let $\boldsymbol{V}_{r, p}=\left\{s_{r-1}+2, \ldots, s_{r}\right\} \times\left\{t_{p-1}+2, \ldots, t_{p}\right\}$ and let $B\left(\boldsymbol{V}_{r, p}\right)$ be its boundary.

To obtain the result it is sufficient to prove that

$$
\mathrm{P}\left(\bigcap_{r, p} M_{\boldsymbol{n}}\left(\boldsymbol{V}_{r, p}\right) \leq u\right) \rightarrow \mathrm{e}^{-v}, \quad v>0
$$

if and only if

$$
\sum_{r, p} \sum_{i \in V_{r, p}} \mathrm{P}\left(B_{i, b_{s}(i)}\right) \rightarrow v>0
$$


This follows from Proposition 2.1, and the relations

$$
\begin{aligned}
\prod_{r, p} \mathrm{P}\left(M_{\boldsymbol{n}}\left(\boldsymbol{V}_{r, p}\right) \leq u\right) & =\exp \left(-(1+o(1)) \sum_{r, p}\left(1-\mathrm{P}\left(M_{\boldsymbol{n}}\left(\boldsymbol{V}_{r, p}\right) \leq u\right)\right)\right) \\
& =\exp \left(-(1+o(1)) \sum_{r, p} \mathrm{P}\left(M_{\boldsymbol{n}}\left(\boldsymbol{V}_{r, p}\right)>u, M_{\boldsymbol{n}}\left(B\left(\boldsymbol{V}_{r, p}\right)\right) \leq u\right)+o(1)\right) \\
& =\exp \left(-(1+o(1)) \sum_{r, p} \sum_{\boldsymbol{i} \in \boldsymbol{V}_{r, p}} \mathrm{P}\left(B_{\boldsymbol{i}, b_{s}(i)}\right)+o(1)\right),
\end{aligned}
$$

since, by (1.1) and (2.1),

$$
\sum_{r, p} \mathrm{P}\left(M_{\boldsymbol{n}}\left(B\left(\boldsymbol{V}_{r, p}\right)\right)>u\right) \leq 2 k_{n_{1}} n_{2} \bar{F}_{\max }+2 k_{n_{2}} n_{1} \bar{F}_{\max }=o(1)
$$

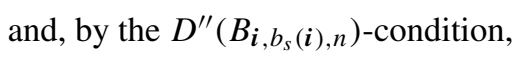

$$
\sum_{r, p} \sum_{\boldsymbol{i}, \boldsymbol{j} \in \boldsymbol{V}_{r, p}} \mathrm{P}\left(B_{\boldsymbol{i}, b_{s}(\boldsymbol{i})}, B_{\boldsymbol{j}, b_{s}(\boldsymbol{j})}\right)=o(1) .
$$

If $\boldsymbol{X}$ is stationary then the result follows by assuming that $u_{\boldsymbol{n}, \boldsymbol{i}}=u_{\boldsymbol{n}}, \boldsymbol{i} \leq \boldsymbol{n}$, and that the

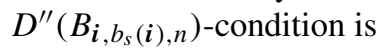

$$
n_{1} n_{2} \sum_{i \leq\left(\left\lfloor n_{1} / k_{n_{1}}\right\rfloor,\left\lfloor n_{2} / k_{n_{2}}\right\rfloor\right)} \mathrm{P}\left(B_{\mathbf{1}, b_{s}(\mathbf{1}), n}, B_{\left.\boldsymbol{i}, b_{s}(\boldsymbol{i}), n\right)} \stackrel{\boldsymbol{n} \rightarrow \infty}{\longrightarrow} 0 .\right.
$$

Weaker local dependence conditions for the stationary case can be considered as in [4].

According to [2], the stationary random field $\boldsymbol{X}$ has extremal index $\theta \in[0,1]$ if, for each $\tau>0$, there exists $\left\{u_{\boldsymbol{n}}^{(\tau)}\right\}_{\boldsymbol{n} \geq \mathbf{1}}$ satisfying (3.2) and $\mathrm{P}\left(M_{\boldsymbol{n}} \leq u_{\boldsymbol{n}}^{(\tau)}\right) \rightarrow \exp (-\theta \tau)$ as $\boldsymbol{n} \rightarrow \infty$. If $\boldsymbol{X}$ is an i.i.d. random field or a stationary random field satisfying the conditions of [11, Proposition 3.1], then the extremal index is equal to 1 .

For nonstationary random fields the extremal index can be defined in a similar way,

$$
\theta(\tau)=\frac{-\log \lim _{n} \mathrm{P}\left(\bigcap_{i \leq n} X_{i} \leq u_{n, i}^{(\tau)}\right)}{\tau},
$$

where

$$
\tau=\lim _{\boldsymbol{n}} \sum_{\boldsymbol{i} \leq \boldsymbol{n}} \mathrm{P}\left(X_{\boldsymbol{i}}>u_{\boldsymbol{n}, \boldsymbol{i}}^{(\tau)}\right) .
$$

Here the extremal index may depend on $\tau$, as pointed out in the examples in [5].

The following result gives a convenient existence criterion for the extremal index and follows immediately from Proposition 3.2.

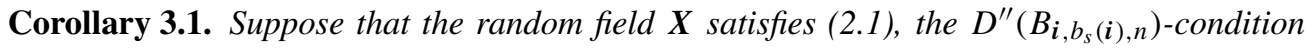
holds for some $s \in\{1, \ldots, 8\}$, and the $D\left(u_{n, i}\right)$-condition holds over $\mathcal{C}\left(B_{i, b_{s}(i), n}\right)$ with $u_{n, i} \equiv$ $u_{\boldsymbol{n}, \boldsymbol{i}}^{(\tau)}$ satisfying (3.3). Then there exists $\theta(\tau)$ if and only if there exists

$$
v=\lim _{n \rightarrow \infty} \sum_{i \leq n} \mathrm{P}\left(B_{i, b_{s}(i), n}\right),
$$


and, in this case, the following expression holds:

$$
\theta(\tau)=\frac{\nu}{\tau}
$$

The extremal index clustering measure can be considered for subfields of $\boldsymbol{X}$. Let $\left\{I_{\boldsymbol{n}}\right\}_{\boldsymbol{n} \geq \mathbf{1}}$ be an increasing sequence of subsets of $R_{n}$. If, for each $\tau>0$, there is a family of levels $\left\{v_{n: i}^{(\tau)}, i \in I_{n}\right\}_{n \geq 1}$ such that

$$
\sum_{i \in I_{n}} \mathrm{P}\left(X_{i}>v_{n, i}^{(\tau)}\right) \stackrel{n \rightarrow \infty}{\longrightarrow} \tau
$$

and

$$
\mathrm{P}\left(\bigcap_{\boldsymbol{i} \in I_{\boldsymbol{n}}} X_{\boldsymbol{i}} \leq v_{\boldsymbol{n}, \boldsymbol{i}}^{(\tau)}\right) \stackrel{\boldsymbol{n} \rightarrow \infty}{\longrightarrow} \exp (-\theta \tau),
$$

we say that $\boldsymbol{X}$ has extremal index $\theta$ over $\bigcup_{n \geq 1} I_{n}$.

In general, we cannot compare the extremal indices over regions with the extremal index of the random field since the normalized levels are not, in general, coincident.

\section{Examples}

Much research has been undertaken, using a specific approach, on the asymptotic behaviour of the maximum of a stationary normal field under a variety of conditions (see [1], [2], [13]). The classical limit still holds under quite a slow rate of dependence decay and the extremal index is equal to 1 . In [12] the authors considered another kind of local dependence condition that restricts the local path behaviour with respect to exceedances in the sense of the $D^{\prime}\left(u_{n}\right)$ condition of [10] and extended the results of [2] to a nonstationary normal field. After some technical lemmas we again found that the extremal index is equal to 1 .

We will now illustrate the results with several 1-dependent random fields which satisfy different local dependence conditions. Let $\boldsymbol{Y}=\left\{Y_{\boldsymbol{n}}\right\}_{\boldsymbol{n} \geq \mathbf{1}}$ be an i.i.d. random field and let $\left\{u_{\boldsymbol{n}}\right\}_{\boldsymbol{n} \geq \mathbf{1}}$ be such that $n_{1} n_{2} \mathrm{P}\left(Y_{\mathbf{1}}>u_{\boldsymbol{n}}\right) \rightarrow \tau$. From $\boldsymbol{Y}$ we shall define several nonstationary and anisotropic random fields.

Let $\boldsymbol{X}=\left\{X_{n}\right\}_{n \geq 1}$ be such that, for each $\boldsymbol{i}=\left(i_{1}, i_{2}\right)=(2 k+1,2 s+1), k, s \geq 0$, the following expressions hold:

$$
\begin{aligned}
X_{i} & =Y_{i}, & X_{b_{1}(i)} & =Y_{b_{1}(i)}, \\
X_{b_{2}(i)} & =\max \left\{Y_{b_{1}(i)}, Y_{b_{2}(i)}\right\}, & X_{b_{3}(i)} & =\max \left\{Y_{i}, Y_{b_{3}(i)}\right\} .
\end{aligned}
$$

This random field only satisfies the $D^{\prime \prime}\left(B_{i, b_{s}(i)}\right)$-condition for $s=3$ and $s=7$ and has extremal index $\theta=\frac{2}{3}$.

Let $\boldsymbol{W}=\left\{W_{\boldsymbol{n}}\right\}_{\boldsymbol{n} \geq \mathbf{1}}$ be such that, for each $\boldsymbol{i}=\left(i_{1}, i_{2}\right)=(2 k+1,2 s+1), k, s \geq 0$, the following expressions hold:

$$
\begin{aligned}
W_{i} & =Y_{i}, & & W_{b_{1}(i)}=\max \left\{Y_{i}, Y_{b_{1}(i)}\right\}, \\
W_{b_{2}(i)} & =Y_{b_{2}(i)}, & & W_{b_{3}(i)}=\max \left\{Y_{\boldsymbol{i}}, Y_{b_{3}(i)}\right\} .
\end{aligned}
$$

This random field satisfies the $D^{\prime \prime}\left(B_{i, b_{s}(i)}\right)$-condition for $s=1, s=3, s=5$, and $s=7$, and has extremal index $\theta=\frac{5}{6}$. 
Finally, let $\boldsymbol{U}=\left\{U_{\boldsymbol{n}}\right\}_{\boldsymbol{n} \geq \mathbf{1}}$ be such that, for each $\boldsymbol{i}=\left(i_{1}, i_{2}\right)=(2 k+1,2 s+1), k, s \geq 0$, the following expressions hold:

$$
\begin{aligned}
U_{i} & =Y_{i}, & & U_{b_{1}(i)}=\max \left\{Y_{\boldsymbol{i}}, Y_{b_{1}(i)}\right\}, \\
U_{b_{2}(i)} & =\max \left\{Y_{b_{1}(i)}, Y_{b_{2}(i)}\right\}, & & U_{b_{3}(i)}=\max \left\{Y_{b_{2}(i)}, Y_{b_{3}(i)}\right\} .
\end{aligned}
$$

This random field does not satisfy the $D^{\prime \prime}\left(B_{i, b_{s}(i)}\right)$-condition. However, it has extremal index $\theta=\frac{2}{3}$, which we can easily compute directly. Let $I_{1, n}=\left\{\left(i_{1}, 2 s+1\right): i_{1} \leq n_{1} \wedge 2 s+1 \leq n_{2}\right\}$ and $I_{2, \boldsymbol{n}}=R_{\boldsymbol{n}}-I_{1, \boldsymbol{n}}$. We have

$$
\mathrm{P}\left(M_{\boldsymbol{n}} \leq u\right)=\mathrm{P}\left(M_{\boldsymbol{n}}\left(I_{1, \boldsymbol{n}}\right) \leq u\right) \mathrm{P}\left(M_{\boldsymbol{n}}\left(I_{2, \boldsymbol{n}}\right) \leq u \mid M_{\boldsymbol{n}}\left(I_{1, \boldsymbol{n}}\right) \leq u\right),
$$

which converges to $\exp \left(-\frac{7}{6}\right) \tau$. Since $\sum_{\boldsymbol{i} \leq \boldsymbol{n}} \mathrm{P}\left(X_{\boldsymbol{i}}>u_{\boldsymbol{n}}\right) \rightarrow \frac{7}{4} \tau>0$, we find that $\theta=\frac{2}{3}$.

For the region $\bigcup_{n \geq 1} I_{1, n}$ we find that $\theta_{I, 1}=\frac{2}{3}$ and for $\bigcup_{n \geq 1} I_{2, n}$ we find that $\theta_{I, 2}=\frac{3}{4}$.

\section{Acknowledgement}

We are grateful to an anonymous referee for corrections and a rigorous report.

\section{References}

[1] AdLER, R. J. (1981). The Geometry of Random Fields. John Wiley, New York.

[2] Chor, H. (2002). Central limit theory and extremes of random fields. Doctoral Thesis, University of North Carolina at Chapel Hill.

[3] Ferreira, H. (1994). Condições de dependência local em teoria de valores extremos. Doctoral Thesis, University of Coimbra.

[4] Ferreira, H. and Pereira, L. (2005). How to compute the extremal index of stationary random fields. Preprint 10/2005, University of Lisbon.

[5] Hüsler, J. (1986). Extreme values of non-stationary random sequences. J. Appl. Prob. 23, 937-950.

[6] Hüsler, J. (1993). A note on exceedances and rare events of non-stationary sequences. J. Appl. Prob. 30, 877-888.

[7] LeAdbetter, M. R. (1983). Extremes and local dependence in stationary sequences. Z. Wahrscheinlichkeitsth. 65, 291-306.

[8] Leadbetter, M. R. and Nandagopalan, S. (1989). On exceedance point processes for stationary sequences under mild oscillation restrictions. In Extreme Value Theory (Lecture Notes Statist. 51), eds J. Hüsler and R.-D. Reiss, Springer, New York, pp. 69-80.

[9] Leadbetter, M. R. And Rootzén, H. (1998). On extreme values in stationary random fields. In Stochastic Processes and Related Topics, Birkhäuser, Boston, MA, pp. 275-285.

[10] Leadbetter, M. R., Lindgren, G. And Rootzén, H. (1983). Extremes and Related Properties of Random Sequences and Processes. Springer, New York.

[11] Pereira, L. and Ferreira, H. (2005). Extremes of quasi-independent random fields and clustering of high values. WSEAS Trans. Math. 4, 333-338.

[12] Pereira, L. And Ferreira, H. (2005). On extreme values in non stationary random fields. Preprint 9/2005, University of Lisbon.

[13] Piterbarg, V. I. (1996). Asymptotic Methods in the Theory of Gaussian Processes and Fields (Trans. Math. Monogr. 148). American Mathematical Society, Providence, RI. 